\title{
Time-reversal of rank-one quantum strategy functions
}

\author{
Yuan $\mathrm{Su}^{1}$ and John Watrous ${ }^{2,3}$ \\ ${ }^{1}$ Department of Computer Science, Institute for Advanced Computer Studies, and Joint Center for Quantum \\ Information and Computer Science, University of Maryland, USA \\ ${ }^{2}$ Institute for Quantum Computing and School of Computer Science, University of Waterloo, Canada \\ ${ }^{3}$ Canadian Institute for Advanced Research, Toronto, Canada \\ October 1, 2018
}

The quantum strategy (or quantum combs) framework is a useful tool for reasoning about interactions among entities that process and exchange quantum information over the course of multiple turns. We prove a time-reversal property for a class of linear functions, defined on quantum strategy representations within this framework, that corresponds to the set of rank-one positive semidefinite operators on a certain space. This time-reversal property states that the maximum value obtained by such a function over all valid quantum strategies is also obtained when the direction of time for the function is reversed, despite the fact that the strategies themselves are generally not time reversible. An application of this fact is an alternative proof of a known relationship between the conditional minand max-entropy of bipartite quantum states, along with generalizations of this relationship.

\section{The quantum strategy framework}

The quantum strategy framework [9], which is also known as the quantum combs framework $[2,4]$, provides a useful framework for reasoning about networks of quantum channels. It may be used to model scenarios in which two or more entities, which we will call players, process and exchange quantum information over the course of multiple rounds of communication; and it is particularly useful when one wishes to consider an optimization over all possible behaviors of one player, for any given specification of the other player or players. Various developments, applications, and variants of the quantum strategy framework can be found in $[1,3,5,8,10]$, for instance, and in a number of other sources.

In the discussion of the quantum strategy framework that follows, as well as in the subsequent sections of this paper, we assume that the reader is familiar with quantum information theory and semidefinite programming. References on this material include $[11,13,15,16]$ as well as [14], which we follow closely with respect to notation and terminology. In particular,

Yuan Su: http://quics.umd.edu/people/yuan-su

John Watrous: https://cs.uwaterloo.ca/ watrous/ 


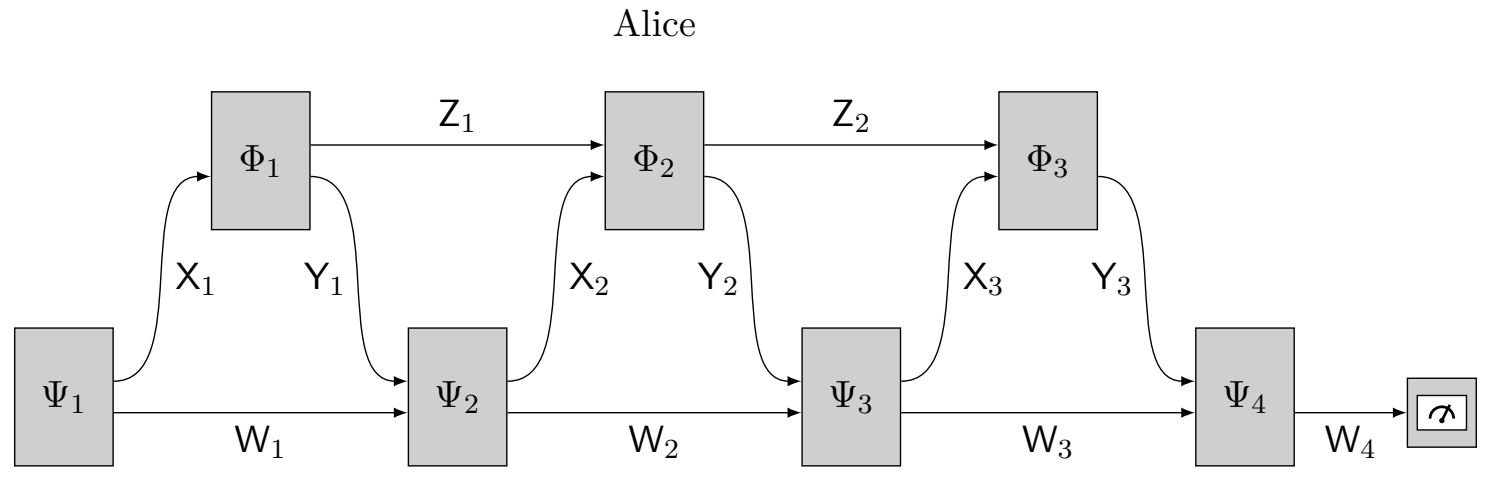

Bob

Figure 1: A six message interaction between Alice and Bob, after which Bob produces a measurement outcome.

we denote quantum registers by capital sans serif letters such as $\mathrm{X}, \mathrm{Y}$, and $\mathrm{Z}$ (sometimes with natural number subscripts), while the same letters (with matching subscripts) in a scripted font, such as $\mathcal{X}, \mathcal{Y}$, and $\mathcal{Z}$ denote the complex Euclidean spaces (i.e., finite-dimensional complex Hilbert spaces) associated with the corresponding registers. The set $\mathrm{L}(\mathcal{X}, \mathcal{Y})$ denotes the set of all linear operators from $\mathcal{X}$ to $\mathcal{Y} ; \mathrm{L}(\mathcal{X})$ is a shorthand for $\mathrm{L}(\mathcal{X}, \mathcal{X}) ; \operatorname{Herm}(\mathcal{X}), \operatorname{Pos}(\mathcal{X})$, $\mathrm{D}(\mathcal{X})$, and $\mathrm{U}(\mathcal{X})$ denote the sets of all Hermitian operators, positive semidefinite operators, density operators, and unitary operators acting on $\mathcal{X} ; \mathrm{C}(\mathcal{X}, \mathcal{Y})$ denotes the set of all channels (i.e., completely positive and trace-preserving maps) mapping $\mathrm{L}(\mathcal{X})$ to $\mathrm{L}(\mathcal{Y})$; and $\mathrm{C}(\mathcal{X})$ is a shorthand for $\mathrm{C}(\mathcal{X}, \mathcal{X})$. The adjoint of an operator $A$ is denoted $A^{*}$, the entry-wise complex conjugate is denoted $\bar{A}$, and the transpose is denoted $A^{\mathrm{T}}$. A similar notation is used for the adjoint and transpose of a channel $\Phi$ (the meaning of which, in the case of the transpose, will be clarified later). The (Hilbert-Schmidt) inner-product is defined as $\langle A, B\rangle=\operatorname{Tr}\left(A^{*} B\right)$ for all operators $A, B \in \mathrm{L}(\mathcal{X})$. Some additional notation will be introduced as it is used.

\section{An example of a six-message interaction}

To explain the aspects of the quantum strategy framework that are relevant to this paper, we will begin by discussing an example of an interaction structure involving six messages exchanged between two players, Alice and Bob. We have chosen to describe a six-message interaction because it is simple and concrete, but nevertheless clearly suggests the underlying structure of an interaction having any finite number of message exchanges. Our main result holds in the general case, which will be considered later, where an arbitrary finite number of message exchanges may take place.

Figure 1 illustrates an interaction between Alice and Bob. In this figure, time proceeds from left to right, and the arrows represent registers either being sent from one player to the other (as is the case for the registers $\mathrm{X}_{1}, \mathrm{Y}_{1}, \mathrm{X}_{2}, \mathrm{Y}_{2}, \mathrm{X}_{3}$, and $\mathrm{Y}_{3}$ ), or momentarily stored by one of the two players (as is the case for $Z_{1}$ and $Z_{2}$, stored by Alice, and $W_{1}, W_{2}, W_{3}, W_{4}$, stored by Bob). Alice's actions are represented by the channels $\Phi_{1}, \Phi_{2}$, and $\Phi_{3}$, and Bob's actions are represented by the channels $\Psi_{1}, \Psi_{2}, \Psi_{3}$, and $\Psi_{4}$, as well as a final measurement, 


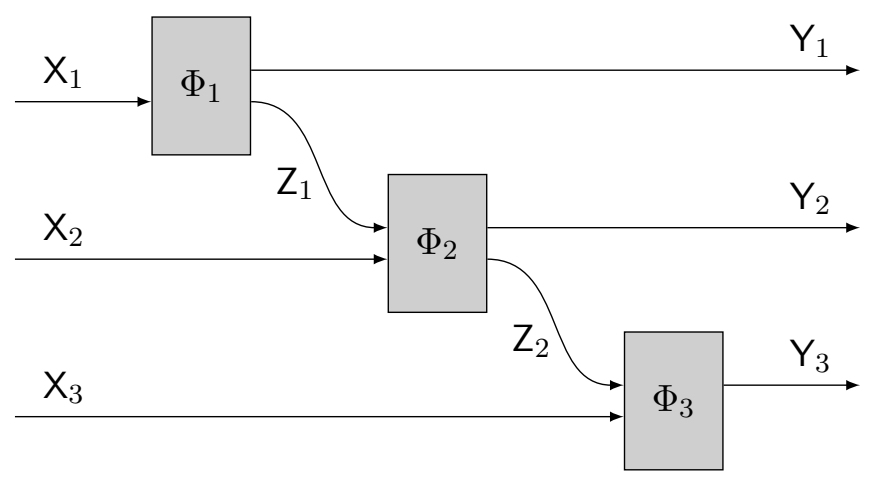

Figure 2: The channel $\Xi_{3}$ that describes Alice's actions in the interaction illustrated in Figure 1.

which is not given a name in the figure.

Suppose that Bob's specification has been fixed, including his choices for the channels $\Psi_{1}$, $\Psi_{2}, \Psi_{3}$, and $\Psi_{4}$, as well as his final measurement, and suppose further that one of Bob's possible measurement outcomes is to be viewed as desirable to Alice. It is then natural to consider an optimization over Alice's possible actions, maximizing the probability that Bob's measurement produces the outcome Alice desires. The quantum strategy framework reveals that this optimization problem can be expressed as a semidefinite program, in the manner that will now be described.

First, a single channel $\Xi_{3}$ that transforms $\left(\mathrm{X}_{1}, \mathrm{X}_{2}, \mathrm{X}_{3}\right)$ to $\left(\mathrm{Y}_{1}, \mathrm{Y}_{2}, \mathrm{Y}_{3}\right)$ is associated with any given choice for Alice's actions. That is, the channel $\Xi_{3}$ takes the form

$$
\Xi_{3} \in \mathrm{C}\left(\mathcal{X}_{1} \otimes \mathcal{X}_{2} \otimes \mathcal{X}_{3}, \mathcal{Y}_{1} \otimes \mathcal{Y}_{2} \otimes \mathcal{Y}_{3}\right)
$$

and for a particular selection of $\Phi_{1}, \Phi_{2}$, and $\Phi_{3}$ may be expressed as

$$
\Xi_{3}=\left(\mathbb{1}_{\mathrm{L}\left(\mathcal{Y}_{1} \otimes \mathcal{Y}_{2}\right)} \otimes \Phi_{3}\right)\left(\mathbb{1}_{\mathrm{L}\left(\mathcal{Y}_{1}\right)} \otimes \Phi_{2} \otimes \mathbb{1}_{\mathrm{L}\left(\mathcal{X}_{3}\right)}\right)\left(\Phi_{1} \otimes \mathbb{1}_{\mathrm{L}\left(\mathcal{X}_{2} \otimes \mathcal{X}_{3}\right)}\right) .
$$

Formally speaking, this composition requires that we view $\Phi_{1}, \Phi_{2}$, and $\Phi_{3}$ as channels of the form $\Phi_{1} \in \mathrm{C}\left(\mathcal{X}_{1}, \mathcal{Y}_{1} \otimes \mathcal{Z}_{1}\right), \Phi_{2} \in \mathrm{C}\left(\mathcal{Z}_{1} \otimes \mathcal{X}_{2}, \mathcal{Y}_{2} \otimes \mathcal{Z}_{2}\right)$, and $\Phi_{3} \in \mathrm{C}\left(\mathcal{Z}_{2} \otimes \mathcal{X}_{3}, \mathcal{Y}_{3}\right)$, as opposed to the forms $\Phi_{1} \in \mathrm{C}\left(\mathcal{X}_{1}, \mathcal{Z}_{1} \otimes \mathcal{Y}_{1}\right), \Phi_{2} \in \mathrm{C}\left(\mathcal{Z}_{1} \otimes \mathcal{X}_{2}, \mathcal{Z}_{2} \otimes \mathcal{Y}_{2}\right)$, and $\Phi_{3} \in \mathrm{C}\left(\mathcal{Z}_{2} \otimes \mathcal{X}_{3}, \mathcal{Y}_{3}\right)$ suggested by Figure 1, so that the ordering of the tensor factors of the various input and output spaces is consistent with the composition. Similar re-orderings of tensor factors should be assumed implicitly throughout this paper as needed. This understanding should not be a source of confusion because we always assign distinct names to distinct registers (and their associated spaces). Figure 2 illustrates the action of the channel $\Xi_{3}$, which in words may be described as the channel obtained if all three of the registers $\left(\mathrm{X}_{1}, \mathrm{X}_{2}, \mathrm{X}_{3}\right)$ are provided initially, and then Alice's actions are composed in the natural way to produce $\left(Y_{1}, Y_{2}, Y_{3}\right)$ as output registers.

It may appear that by considering the channel $\Xi_{3}$, one is ignoring the possibility that Bob's actions could, for instance, allow the contents of $Y_{1}$ or $Y_{2}$ to influence what is input into $X_{2}$ or $X_{3}$. Despite this appearance, the influence that Alice's actions have from the viewpoint of Bob, including the probability for each of his measurement outcomes to appear, is uniquely determined by the channel $\Xi_{3}$.

Naturally, not all channels of the form (1) will arise from a composition of channels $\Phi_{1}$, $\Phi_{2}$, and $\Phi_{3}$ as in (2); the fact that $\Phi_{1}$ is effectively performed first, $\Phi_{2}$ is performed second, 
and $\Phi_{3}$ is performed third imposes constraints on the channels $\Xi_{3}$ that can be obtained. In particular, consider the channel that results when $\Xi_{3}$ is performed and then the partial trace is performed on $\mathcal{Y}_{3}$. As $\Phi_{3}$ is a channel, discarding its output is equivalent to discarding its inputs, from which it follows that

$$
\operatorname{Tr}_{\mathcal{Y}_{3}} \circ \Xi_{3}=\Xi_{2} \circ \operatorname{Tr}_{\mathcal{X}_{3}}
$$

where the circles represent channel compositions and $\Xi_{2} \in \mathrm{C}\left(\mathcal{X}_{1} \otimes \mathcal{X}_{2}, \mathcal{Y}_{1} \otimes \mathcal{Y}_{2}\right)$ is the channel defined as

$$
\Xi_{2}=\left(\mathbb{1}_{\mathrm{L}\left(\mathcal{Y}_{1}\right)} \otimes\left(\operatorname{Tr}_{\mathcal{Z}_{2}} \circ \Phi_{2}\right)\right)\left(\Phi_{1} \otimes \mathbb{1}_{\mathrm{L}\left(\mathcal{X}_{2}\right)}\right) .
$$

That is, $\Xi_{2}$ is the channel obtained from $\Phi_{1}$ and $\Phi_{2}$, followed by the partial trace over $\mathcal{Z}_{2}$, by a similar process to the one used to obtain $\Xi_{3}$. By similar reasoning, one finds that

$$
\operatorname{Tr}_{\mathcal{Y}_{2}} \circ \Xi_{2}=\Xi_{1} \circ \operatorname{Tr}_{\mathcal{X}_{2}}
$$

where $\Xi_{1} \in \mathrm{C}\left(\mathcal{X}_{1}, \mathcal{Y}_{1}\right)$ is the channel given by $\Xi_{1}=\operatorname{Tr}_{\mathcal{Z}_{1}} \circ \Phi_{1}$.

Somewhat remarkably, this is not only a necessary condition on the channel $\Xi_{3}$, but also a sufficient one, for it to be obtained from a composition of channels $\Phi_{1}, \Phi_{2}$, and $\Phi_{3}$ as described above. That is, given any channel

$$
\Xi_{3} \in \mathrm{C}\left(\mathcal{X}_{1} \otimes \mathcal{X}_{2} \otimes \mathcal{X}_{3}, \mathcal{Y}_{1} \otimes \mathcal{Y}_{2} \otimes \mathcal{Y}_{3}\right)
$$

satisfying (3) and (5), for some choice of channels

$$
\begin{gathered}
\Xi_{2} \in \mathrm{C}\left(\mathcal{X}_{1} \otimes \mathcal{X}_{2}, \mathcal{Y}_{1} \otimes \mathcal{Y}_{2}\right), \\
\Xi_{1} \in \mathrm{C}\left(\mathcal{X}_{1}, \mathcal{Y}_{1}\right),
\end{gathered}
$$

there must exist channels

$$
\begin{gathered}
\Phi_{1} \in \mathrm{C}\left(\mathcal{X}_{1}, \mathcal{Y}_{1} \otimes \mathcal{Z}_{1}\right), \\
\Phi_{2} \in \mathrm{C}\left(\mathcal{Z}_{1} \otimes \mathcal{X}_{2}, \mathcal{Y}_{2} \otimes \mathcal{Z}_{2}\right), \\
\Phi_{3} \in \mathrm{C}\left(\mathcal{Z}_{2} \otimes \mathcal{X}_{3}, \mathcal{Y}_{3}\right),
\end{gathered}
$$

for spaces $\mathcal{Z}_{1}$ and $\mathcal{Z}_{2}$ having sufficiently large dimension, so that (2) holds. This fact is proved in $[2,4,9]$, and we note that a key idea through which this equivalence is proved may be found in [7].

The next step toward an expression of the optimization problem suggested above as a semidefinite program makes use of the Choi representation of channels. The Choi representation of the channel $\Xi_{3}$ takes the form

$$
J\left(\Xi_{3}\right) \in \operatorname{Pos}\left(\mathcal{Y}_{1} \otimes \mathcal{Y}_{2} \otimes \mathcal{Y}_{3} \otimes \mathcal{X}_{1} \otimes \mathcal{X}_{2} \otimes \mathcal{X}_{3}\right)
$$

as the complete positivity of $\Phi_{1}, \Phi_{2}$, and $\Phi_{3}$ implies that $\Xi_{3}$ is also completely positive, and therefore $J\left(\Xi_{3}\right)$ is positive semidefinite. The constraints on the channel $\Xi_{3}$ described previously correspond (conveniently) to linear constraints; one has that (3) and (5) hold, for some choice of channels $\Xi_{2}$ and $\Xi_{1}$, if and only if the Choi representation $X_{3}=J\left(\Xi_{3}\right)$ of $\Xi_{3}$ satisfies

$$
\begin{gathered}
\operatorname{Tr}_{\mathcal{Y}_{3}}\left(X_{3}\right)=X_{2} \otimes \mathbb{1}_{\mathcal{X}_{3}}, \\
\operatorname{Tr}_{\mathcal{Y}_{2}}\left(X_{2}\right)=X_{1} \otimes \mathbb{1}_{\mathcal{X}_{2}}, \\
\operatorname{Tr}_{\mathcal{Y}_{1}}\left(X_{1}\right)=\mathbb{1}_{\mathcal{X}_{1}},
\end{gathered}
$$


for some choice of operators

$$
\begin{gathered}
X_{2} \in \operatorname{Pos}\left(\mathcal{Y}_{1} \otimes \mathcal{Y}_{2} \otimes \mathcal{X}_{1} \otimes \mathcal{X}_{2}\right), \\
X_{1} \in \operatorname{Pos}\left(\mathcal{Y}_{1} \otimes \mathcal{X}_{1}\right) .
\end{gathered}
$$

These operators correspond to the Choi representations $X_{2}=J\left(\Xi_{2}\right)$ and $X_{1}=J\left(\Xi_{1}\right)$.

Finally, the probability that Bob's measurement produces a given outcome is a linear function of the channel $\Xi_{3}$, and is therefore a linear function of the Choi representation $X_{3}=$ $J\left(\Xi_{3}\right)$. Although this process is not relevant to the main result of this paper, we note that it is possible to obtain an explicit description of this linear function given a specification of Bob's actions, including his final measurement. In somewhat vague terms, the linear function describing Bob's probability to produce a particular measurement outcome is given by $\left\langle P, X_{3}\right\rangle$, where

$$
P \in \operatorname{Pos}\left(\mathcal{Y}_{1} \otimes \mathcal{Y}_{2} \otimes \mathcal{Y}_{3} \otimes \mathcal{X}_{1} \otimes \mathcal{X}_{2} \otimes \mathcal{X}_{3}\right)
$$

is an operator that is obtained from $\Psi_{1}, \Psi_{2}, \Psi_{3}, \Psi_{4}$, and the measurement operator corresponding to the outcome being considered by a process very similar to the one through which $X_{3}$ is obtained from $\Phi_{1}, \Phi_{2}$, and $\Phi_{3}$. The reader is again referred to [2, 4, 9] for further details.

More generally, an arbitrary real-valued linear function of the operator $X_{3}$ may be expressed as $\left\langle H, X_{3}\right\rangle$ for some choice of a Hermitian operator

$$
H \in \operatorname{Herm}\left(\mathcal{Y}_{1} \otimes \mathcal{Y}_{2} \otimes \mathcal{Y}_{3} \otimes \mathcal{X}_{1} \otimes \mathcal{X}_{2} \otimes \mathcal{X}_{3}\right)
$$

which need not represent the probability with which a particular measurement outcome is obtained for channels $\Psi_{1}, \ldots, \Psi_{4}$ followed by a measurement. Such a function could, for instance, represent an expected payoff for Alice's actions, under the assumption that a real-valued payoff is associated with each of Bob's measurement outcomes.

\section{General semidefinite programming formulation}

As mentioned previously, the six-message example just described generalizes to any finite number of message exchanges. If the number of message exchanges is equal to $n$, the input registers to Alice (the player whose actions are being optimized) are $\mathrm{X}_{1}, \ldots, \mathrm{X}_{n}$, and the

output registers of Alice are $\mathrm{Y}_{1}, \ldots, \mathrm{Y}_{n}$, then the possible strategies for Alice are represented by channels of the form

$$
\Xi_{n} \in \mathrm{C}\left(\mathcal{X}_{1} \otimes \cdots \otimes \mathcal{X}_{n}, \mathcal{Y}_{1} \otimes \cdots \otimes \mathcal{Y}_{n}\right)
$$

that obey constraints that generalize (3) and (5). Specifically, there must exist channels

$$
\begin{gathered}
\Xi_{n-1} \in \mathrm{C}\left(\mathcal{X}_{1} \otimes \cdots \otimes \mathcal{X}_{n-1}, \mathcal{Y}_{1} \otimes \cdots \otimes \mathcal{Y}_{n-1}\right) \\
\vdots \\
\Xi_{1} \in \mathrm{C}\left(\mathcal{X}_{1}, \mathcal{Y}_{1}\right)
\end{gathered}
$$

such that

$$
\operatorname{Tr}_{\mathcal{Y}_{k}} \circ \Xi_{k}=\Xi_{k-1} \circ \operatorname{Tr}_{\mathcal{X}_{k}}
$$




\section{Primal problem}

$$
\begin{aligned}
\operatorname{maximize}: & \left\langle H, X_{n}\right\rangle \\
\text { subject to: } & \operatorname{Tr}_{\mathcal{Y}_{n}}\left(X_{n}\right)=X_{n-1} \otimes \mathbb{1}_{\mathcal{X}_{n}}, \\
& \vdots \\
& \operatorname{Tr}_{\mathcal{Y}_{2}}\left(X_{2}\right)=X_{1} \otimes \mathbb{1}_{\mathcal{X}_{2}}, \\
& \operatorname{Tr}_{\mathcal{Y}_{1}}\left(X_{1}\right)=\mathbb{1}_{\mathcal{X}_{1}}, \\
& X_{n} \in \operatorname{Pos}\left(\mathcal{Y}_{1} \otimes \cdots \otimes \mathcal{Y}_{n} \otimes \mathcal{X}_{1} \otimes \cdots \otimes \mathcal{X}_{n}\right), \\
& \vdots \\
& X_{2} \in \operatorname{Pos}\left(\mathcal{Y}_{1} \otimes \mathcal{Y}_{2} \otimes \mathcal{X}_{1} \otimes \mathcal{X}_{2}\right), \\
& X_{1} \in \operatorname{Pos}\left(\mathcal{Y}_{1} \otimes \mathcal{X}_{1}\right) .
\end{aligned}
$$

\section{Dual problem}

$$
\begin{aligned}
\operatorname{minimize}: & \operatorname{Tr}\left(Y_{1}\right) \\
\text { subject to: } & Y_{n} \otimes \mathbb{1}_{\mathcal{Y}_{n} \geq H,} \\
& Y_{n-1} \otimes \mathbb{1}_{\mathcal{Y}_{n-1}} \geq \operatorname{Tr}_{\mathcal{X}_{n}}\left(Y_{n}\right), \\
& \vdots \\
& Y_{1} \otimes \mathbb{1}_{\mathcal{Y}_{1}} \geq \operatorname{Tr}_{\mathcal{X}_{2}}\left(Y_{2}\right), \\
& Y_{n} \in \operatorname{Herm}\left(\mathcal{Y}_{1} \otimes \cdots \otimes \mathcal{Y}_{n-1} \otimes \mathcal{X}_{1} \otimes \cdots \otimes \mathcal{X}_{n}\right), \\
& Y_{n-1} \in \operatorname{Herm}\left(\mathcal{Y}_{1} \otimes \cdots \otimes \mathcal{Y}_{n-2} \otimes \mathcal{X}_{1} \otimes \cdots \otimes \mathcal{X}_{n-1}\right), \\
& \vdots \\
& Y_{1} \in \operatorname{Herm}\left(\mathcal{X}_{1}\right) .
\end{aligned}
$$

Figure 3: The semidefinite program representing a maximization of a linear function of an $n$-turn strategy.

for all $k \in\{2, \ldots, n\}$. For the maximization of a real-valued linear function over all strategies for Alice, represented by a Hermitian operator

$$
H \in \operatorname{Herm}\left(\mathcal{Y}_{1} \otimes \cdots \otimes \mathcal{Y}_{n} \otimes \mathcal{X}_{1} \otimes \cdots \otimes \mathcal{X}_{n}\right),
$$

one obtains the semidefinite program described in Figure 3. The primal problem corresponds to an optimization over all Choi representations of the channels $\Xi_{1}, \ldots, \Xi_{n}$. This semidefinite programming formulation is implicit in [9], and first appeared explicitly in [8]. It also appears in [1], where it was used to define a generalized notion of min-entropy for quantum networks.

It may be noted that the general problem just formulated concerns interactions involving an even number of register exchanges, where Alice (the player whose actions are being optimized) always receives the first transmission, represented by $\mathrm{X}_{1}$, and sends the last transmission, represented by $Y_{n}$. However, one is free to take either or both of the registers $X_{1}$ and $Y_{n}$ to be trivial registers, so that correspondingly $\mathcal{X}_{1}=\mathbb{C}$ and $/$ or $\mathcal{Y}_{n}=\mathbb{C}$. This is tantamount to allowing either an odd number of register exchanges or an even number in the situation that 
Alice sends the first (nontrivial) register and receives the last.

\section{Statement and proof of the main result}

The main result of the current paper concerns the optimization problem described in the previous section, as represented by the semidefinite program in Figure 3, in the case that $H=u u^{*}$ is a rank one positive semidefinite operator. The result to be described does not hold in general when $H$ does not take this form.

In order to explain the main result in precise terms, it will be helpful to introduce some notation. Suppose that a positive integer $n$ along with spaces $\mathcal{X}_{1}, \ldots, \mathcal{X}_{n}$ and $\mathcal{Y}_{1}, \ldots, \mathcal{Y}_{n}$ have been fixed. For each $k \in\{1, \ldots, n\}$, let

$$
\mathcal{S}_{k}\left(\mathcal{X}_{1}, \ldots, \mathcal{X}_{k} ; \mathcal{Y}_{1}, \ldots, \mathcal{Y}_{k}\right) \subset \operatorname{Pos}\left(\mathcal{Y}_{1} \otimes \cdots \otimes \mathcal{Y}_{k} \otimes \mathcal{X}_{1} \otimes \cdots \otimes \mathcal{X}_{k}\right)
$$

denote the primal-feasible choices for the operator $X_{k}$ in the semidefinite program specified in Figure 3. That is, we define

$$
\mathcal{S}_{1}\left(\mathcal{X}_{1} ; \mathcal{Y}_{1}\right)=\left\{X_{1} \in \operatorname{Pos}\left(\mathcal{Y}_{1} \otimes \mathcal{X}_{1}\right): \operatorname{Tr}_{\mathcal{Y}_{1}}\left(X_{1}\right)=\mathbb{1}_{\mathcal{X}_{1}}\right\}
$$

(which is the set of all Choi operators of channels of the form $\Xi_{1} \in \mathrm{C}\left(\mathcal{X}_{1}, \mathcal{Y}_{1}\right)$ ), and

$$
\begin{aligned}
& \mathcal{S}_{k}\left(\mathcal{X}_{1}, \ldots, \mathcal{X}_{k} ; \mathcal{Y}_{1}, \ldots, \mathcal{Y}_{k}\right) \\
& =\left\{X_{k} \in \operatorname{Pos}\left(\mathcal{Y}_{1} \otimes \cdots \otimes \mathcal{Y}_{k} \otimes \mathcal{X}_{1} \otimes \cdots \otimes \mathcal{X}_{k}\right): \operatorname{Tr}_{Y_{k}}\left(X_{k}\right)=X_{k-1} \otimes \mathbb{1}_{\mathcal{X}_{k}}\right. \\
& \left.\quad \text { for some } X_{k-1} \in \mathcal{S}_{k-1}\left(\mathcal{X}_{1}, \ldots, \mathcal{X}_{k-1} ; \mathcal{Y}_{1}, \ldots, \mathcal{Y}_{k-1}\right)\right\}
\end{aligned}
$$

for $k \in\{2, \ldots, n\}$. The primal form of the semidefinite program described in Figure 3 can therefore be expressed succinctly as

$$
\begin{aligned}
\operatorname{maximize}: & \langle H, X\rangle \\
\text { subject to: } & X \in \mathcal{S}_{n}\left(\mathcal{X}_{1}, \ldots, \mathcal{X}_{n} ; \mathcal{Y}_{1}, \ldots, \mathcal{Y}_{n}\right) .
\end{aligned}
$$

We will refer to operators in the sets defined above as strategy operators, as they represent $n$-turn strategies with respect to the quantum strategy framework.

Let us also define an isometry

$$
W \in \mathrm{U}\left(\mathcal{Y}_{1} \otimes \cdots \otimes \mathcal{Y}_{n} \otimes \mathcal{X}_{1} \otimes \cdots \otimes \mathcal{X}_{n}, \mathcal{X}_{n} \otimes \cdots \otimes \mathcal{X}_{1} \otimes \mathcal{Y}_{n} \otimes \cdots \otimes \mathcal{Y}_{1}\right)
$$

by the action

$$
W\left(y_{1} \otimes \cdots \otimes y_{n} \otimes x_{1} \otimes \cdots \otimes x_{n}\right)=x_{n} \otimes \cdots \otimes x_{1} \otimes y_{n} \otimes \cdots \otimes y_{1}
$$

for all vectors $x_{1} \in \mathcal{X}_{1}, \ldots, x_{n} \in \mathcal{X}_{n}$ and $y_{1} \in \mathcal{Y}_{1}, \ldots, y_{n} \in \mathcal{Y}_{n}$. In words, $W$ simply reverses the order of the tensor factors of the space $\mathcal{Y}_{1} \otimes \cdots \otimes \mathcal{Y}_{n} \otimes \mathcal{X}_{1} \otimes \cdots \otimes \mathcal{X}_{n}$, yielding a vector in $\mathcal{X}_{n} \otimes \cdots \otimes \mathcal{X}_{1} \otimes \mathcal{Y}_{n} \otimes \cdots \otimes \mathcal{Y}_{1}$ that, aside from this re-ordering of tensor factors, is the same as its input vector. 


\section{Statement of the main result}

With the notation just introduced in hand, the main theorem may now be stated.

Theorem 1. Let $\mathcal{X}_{1}, \ldots, \mathcal{X}_{n}$ and $\mathcal{Y}_{1}, \ldots, \mathcal{Y}_{n}$ be complex Euclidean spaces, for $n$ a positive integer, let

$$
u \in \mathcal{Y}_{1} \otimes \cdots \otimes \mathcal{Y}_{n} \otimes \mathcal{X}_{1} \otimes \cdots \otimes \mathcal{X}_{n}
$$

be a vector, and let

$$
X \in \mathcal{S}_{n}\left(\mathcal{X}_{1}, \ldots, \mathcal{X}_{n} ; \mathcal{Y}_{1}, \ldots, \mathcal{Y}_{n}\right)
$$

be a strategy operator. There exists a strategy operator

$$
Y \in \mathcal{S}_{n}\left(\mathcal{Y}_{n}, \ldots, \mathcal{Y}_{1} ; \mathcal{X}_{n}, \ldots, \mathcal{X}_{1}\right)
$$

such that

$$
\left\langle W u u^{*} W^{*}, Y\right\rangle \geq\left\langle u u^{*}, X\right\rangle .
$$

If it is the case that $\operatorname{dim}\left(\mathcal{Y}_{1} \otimes \cdots \otimes \mathcal{Y}_{n}\right) \leq \operatorname{dim}\left(\mathcal{X}_{1} \otimes \cdots \otimes \mathcal{X}_{n}\right)$, then the operator $Y$ may be chosen so that equality holds in (27).

Corollary 2. Let $\mathcal{X}_{1}, \ldots, \mathcal{X}_{n}$ and $\mathcal{Y}_{1}, \ldots, \mathcal{Y}_{n}$ be complex Euclidean spaces, for $n$ a positive integer, and let

$$
u \in \mathcal{Y}_{1} \otimes \cdots \otimes \mathcal{Y}_{n} \otimes \mathcal{X}_{1} \otimes \cdots \otimes \mathcal{X}_{n}
$$

be a vector. The semidefinite optimization problems

$$
\begin{array}{ll}
\text { maximize: } & \left\langle u u^{*}, X\right\rangle \\
\text { subject to: } & X \in \mathcal{S}_{n}\left(\mathcal{X}_{1}, \ldots, \mathcal{X}_{n} ; \mathcal{Y}_{1}, \ldots, \mathcal{Y}_{n}\right)
\end{array}
$$

and

$$
\begin{array}{ll}
\text { maximize: } & \left\langle W u u^{*} W^{*}, Y\right\rangle \\
\text { subject to: } & Y \in \mathcal{S}_{n}\left(\mathcal{Y}_{n}, \ldots, \mathcal{Y}_{1} ; \mathcal{X}_{n}, \ldots, \mathcal{X}_{1}\right)
\end{array}
$$

have the same optimum value.

Remark. Using the notation introduced in [1], which defines a quantum network generalization of conditional min-entropy, the equivalence expressed by Corollary 2 may alternatively be written

$$
\begin{gathered}
\mathrm{H}_{\min }\left(\mathrm{Y}_{n} \mid \mathrm{X}_{1}, \mathrm{Y}_{1}, \ldots, \mathrm{X}_{n-1}, \mathrm{Y}_{n-1}, \mathrm{X}_{n}\right)_{u u^{*}} \\
=\mathrm{H}_{\min }\left(\mathrm{X}_{1} \mid \mathrm{Y}_{n}, \mathrm{X}_{n}, \ldots, \mathrm{Y}_{2}, \mathrm{X}_{2}, \mathrm{Y}_{1}\right)_{u u^{*}}
\end{gathered}
$$

for every vector $u \in \mathcal{X}_{1} \otimes \mathcal{Y}_{1} \otimes \cdots \otimes \mathcal{X}_{n} \otimes \mathcal{Y}_{n}$.

Interpretations of the main theorem

Theorem 1 establishes a time-reversal property of rank-one strategy functions. Intuitively speaking, the linear function

$$
Y \mapsto\left\langle W u u^{*} W^{*}, Y\right\rangle
$$

defined on $\mathcal{S}_{n}\left(\mathcal{Y}_{n}, \ldots, \mathcal{Y}_{1} ; \mathcal{X}_{n}, \ldots, \mathcal{X}_{1}\right)$ represents the time-reversal of the linear function

$$
X \mapsto\left\langle u u^{*}, X\right\rangle
$$


defined on $\mathcal{S}_{n}\left(\mathcal{X}_{1}, \ldots, \mathcal{X}_{n} ; \mathcal{Y}_{1}, \ldots, \mathcal{Y}_{n}\right)$, in the sense that the two functions differ only in the reversal of the ordering of the register exchanges: $\mathrm{X}_{1}, \mathrm{Y}_{1}, \ldots, \mathrm{X}_{n}, \mathrm{Y}_{n}$ for the function corresponding to $u u^{*}$ and $\mathrm{Y}_{n}, \mathrm{X}_{n}, \ldots, \mathrm{Y}_{1}, \mathrm{X}_{1}$ for the function corresponding to $W u u^{*} W^{*}$.

For a given choice of $X \in \mathcal{S}_{n}\left(\mathcal{X}_{1}, \ldots, \mathcal{X}_{n} ; \mathcal{Y}_{1}, \ldots, \mathcal{Y}_{n}\right)$, it is generally not the case that $W^{*} X W \in \mathcal{S}_{n}\left(\mathcal{Y}_{n}, \ldots, \mathcal{Y}_{1} ; \mathcal{X}_{n}, \ldots, \mathcal{X}_{1}\right)$. It may not even be the case that $W^{*} X W$ is the Choi representation of a channel, and in the case that $W^{*} X W$ is the Choi representation of a channel, it will generally not be the case that this channel obeys the constraints necessary for it to be a valid strategy operator. When combined with the observation that $\mathcal{S}_{n}\left(\mathcal{X}_{1}, \ldots, \mathcal{X}_{n} ; \mathcal{Y}_{1}, \ldots, \mathcal{Y}_{n}\right)$ and $\mathcal{S}_{n}\left(\mathcal{Y}_{n}, \ldots, \mathcal{Y}_{1} ; \mathcal{X}_{n}, \ldots, \mathcal{X}_{1}\right)$ are compact and convex sets, this fact implies that the main theorem cannot possibly hold for all Hermitian operators $H$ by the separating hyperplane theorem. For small values of $n$ and for spaces having small dimensions, simple examples of operators $H$ for which the main theorem fails may also easily be obtained through random selections.

In Section 4 we discuss another interpretation of Theorem 1, which concerns multiple round entanglement manipulation.

\section{Proof of Theorem 1}

We will now prove Theorem 1 . The first step is to express the strategy represented by $X$ as a sequence of channels corresponding to invertible isometries (i.e., unitary operators for which the input and output spaces have different names but necessarily the same dimension), assuming an auxiliary input space initialized to a pure state is made available.

Through the repeated application of the Stinespring dilation theorem, together with the result of $[2,4,9]$ establishing that $X=J\left(\Xi_{n}\right)$ is the Choi representation of a channel $\Xi_{n}$ arising from a valid $n$-turn strategy, one finds that there must exist complex Euclidean spaces $\mathcal{Z}_{0}, \ldots, \mathcal{Z}_{n}$ satisfying $\operatorname{dim}\left(\mathcal{Z}_{k-1} \otimes \mathcal{X}_{k}\right)=\operatorname{dim}\left(\mathcal{Z}_{k} \otimes \mathcal{Y}_{k}\right)$ for all $k \in\{1, \ldots, n\}$, a unit vector $v \in \mathcal{Z}_{0}$, and invertible isometries $U_{1}, \ldots, U_{n}$ of the form

$$
U_{k} \in \mathrm{U}\left(\mathcal{Z}_{k-1} \otimes \mathcal{X}_{k}, \mathcal{Y}_{k} \otimes \mathcal{Z}_{k}\right)
$$

such that

$$
\Xi_{n}(Z)=\operatorname{Tr}_{\mathcal{Z}_{n}}\left(U\left(v v^{*} \otimes Z\right) U^{*}\right)
$$

for all $Z \in \mathrm{L}\left(\mathcal{X}_{1} \otimes \cdots \otimes \mathcal{X}_{n}\right)$, where

$$
\begin{aligned}
U & =\left(\mathbb{1}_{\mathcal{Y}_{1} \otimes \cdots \otimes \mathcal{Y}_{n-1}} \otimes U_{n}\right) \cdots\left(U_{1} \otimes \mathbb{1}_{\mathcal{X}_{2} \otimes \cdots \otimes \mathcal{X}_{n}}\right) \\
& \in \mathrm{U}\left(\mathcal{Z}_{0} \otimes \mathcal{X}_{1} \otimes \cdots \otimes \mathcal{X}_{n}, \mathcal{Y}_{1} \otimes \cdots \otimes \mathcal{Y}_{n} \otimes \mathcal{Z}_{n}\right)
\end{aligned}
$$

In words, the strategy represented by the operator $X$ is implemented by first initializing a register $\mathrm{Z}_{0}$ to the pure state $v$, then applying the invertible isometric channels corresponding to $U_{1}, \ldots, U_{n}$, and finally discarding $Z_{n}$ after the interaction has finished. (The top picture in Figure 4 illustrates this for the case $n=3$.)

The vector $u$ may be expressed as

$$
u=\sum_{\substack{a_{1}, \ldots, a_{n} \\ b_{1}, \ldots, b_{n}}} u\left(b_{1}, \ldots, b_{n}, a_{1}, \ldots, a_{n}\right)\left|b_{1}\right\rangle \cdots\left|b_{n}\right\rangle\left|a_{1}\right\rangle \cdots\left|a_{n}\right\rangle,
$$



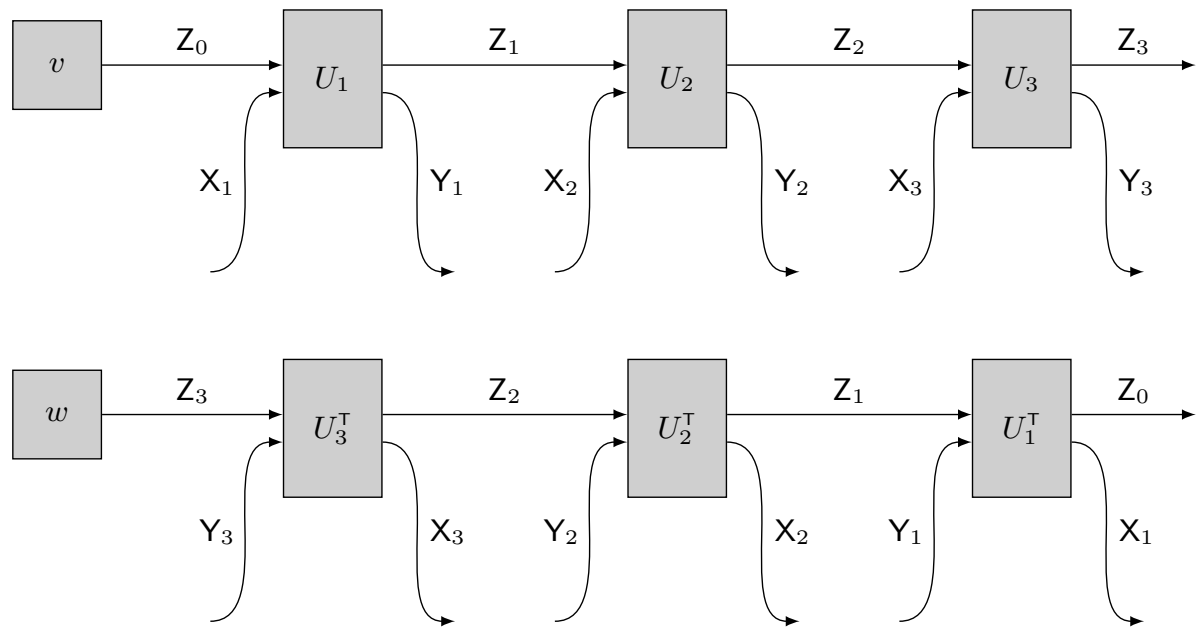

Figure 4: An arbitrary strategy may be implemented by initializing a register $\mathrm{Z}_{0}$ to a pure state $v$, followed by the application of an invertible isometric channel on each turn, and finally by discarding the last memory register $Z_{n}$ (which is $Z_{3}$ in the picture). The time-reversed strategy whose existence is implied by the main theorem is obtained by setting the register $Z_{n}\left(Z_{3}\right.$ in the picture) to an appropriate choice of a pure state $w$, followed by the application of invertible isometric channels obtained by transposing the original isometries, and finally by discarding the memory register $Z_{0}$.

where the sum is over all standard basis states $\left|a_{1}\right\rangle, \ldots,\left|a_{n}\right\rangle$ of $\mathcal{X}_{1}, \ldots, \mathcal{X}_{n}$ and $\left|b_{1}\right\rangle, \ldots,\left|b_{n}\right\rangle$ of $\mathcal{Y}_{1}, \ldots, \mathcal{Y}_{n}$, respectively. Based on this expression, define an operator $A \in \mathrm{L}\left(\mathcal{Z}_{0}, \mathcal{Z}_{n}\right)$ as

$$
\begin{array}{r}
A=\sum_{\substack{a_{1}, \ldots, a_{n} \\
b_{1}, \ldots, b_{n}}} u\left(b_{1}, \ldots, b_{n}, a_{1}, \ldots, a_{n}\right)\left(\left\langle b_{n}\right| \otimes \mathbb{1}_{\mathcal{Z}_{n}}\right) U_{n}\left(\mathbb{1}_{\mathcal{Z}_{n-1}} \otimes\left|a_{n}\right\rangle\right) \\
\cdots\left(\left\langle b_{1}\right| \otimes \mathbb{1}_{\mathcal{Z}_{1}}\right) U_{1}\left(\mathbb{1}_{\mathcal{Z}_{0}} \otimes\left|a_{1}\right\rangle\right) .
\end{array}
$$

By considering the action of the strategy represented by $v$ and $U_{1}, \ldots, U_{n}$, then performing the required operator-vector multiplications required to evaluate the expression $\left\langle u u^{*}, X\right\rangle$ when $X=J\left(\Xi_{n}\right)$ for $\Xi_{n}$ given by $(35)$, one concludes that

$$
\left\langle u u^{*}, X\right\rangle=\|A v\|^{2}=\left\langle v v^{*}, A^{*} A\right\rangle .
$$

Next we turn to the reversed interaction. To obtain a strategy operator $Y$ satisfying the requirements of the theorem, we consider the strategy obtained by initializing the register $Z_{n}$ to a particular choice of a pure state $w$, which will be selected later, then applying in sequence the invertible isometric channels corresponding to the operators $U_{n}^{\top}, \ldots, U_{1}^{\top}$. (The bottom picture in Figure 4 illustrates this for the case $n=3$.) That is, for

$$
\begin{aligned}
V & =\left(\mathbb{1}_{\mathcal{X}_{n} \otimes \cdots \otimes \mathcal{X}_{2}} \otimes U_{1}^{\top}\right) \cdots\left(U_{n}^{\top} \otimes \mathbb{1}_{\mathcal{Y}_{n-1} \otimes \cdots \otimes \mathcal{Y}_{1}}\right) \\
& \in \mathrm{U}\left(\mathcal{Z}_{n} \otimes \mathcal{Y}_{n} \otimes \cdots \otimes \mathcal{Y}_{1}, \mathcal{Z}_{0} \otimes \mathcal{X}_{n} \otimes \cdots \otimes \mathcal{X}_{1}\right)
\end{aligned}
$$

we consider the channel $\Theta_{n} \in \mathrm{C}\left(\mathcal{Y}_{n} \otimes \cdots \otimes \mathcal{Y}_{1}, \mathcal{X}_{n} \otimes \cdots \otimes \mathcal{X}_{1}\right)$ defined as

$$
\Theta_{n}(Z)=\operatorname{Tr}_{\mathcal{Z}_{0}}\left(V\left(w w^{*} \otimes Z\right) V^{*}\right)
$$


for all $Z \in \mathrm{L}\left(\mathcal{Y}_{n} \otimes \cdots \otimes \mathcal{Y}_{1}\right)$. It is evident from the specification of this channel, irrespective of the choice of the pure state $w$, that $Y=J\left(\Theta_{n}\right) \in \mathcal{S}_{n}\left(\mathcal{Y}_{n}, \ldots, \mathcal{Y}_{1} ; \mathcal{X}_{n}, \ldots, \mathcal{X}_{1}\right)$. By considering the action of this strategy, a similar calculation to the one above reveals that

$$
\left\langle W u u^{*} W^{*}, Y\right\rangle=\left\|A^{\top} w\right\|^{2}=\left\langle w w^{*}, \bar{A} A^{\top}\right\rangle .
$$

The nonzero eigenvalues of $A^{*} A$ and $\bar{A} A^{\top}$ are equal, and therefore by choosing $w$ to be an eigenvector corresponding to the largest eigenvalue of $\bar{A} A^{\top}$ one obtains

$$
\left\langle W u u^{*} W^{*}, Y\right\rangle=\left\langle w w^{*}, \bar{A} A^{\top}\right\rangle \geq\left\langle v v^{*}, A^{*} A\right\rangle=\left\langle u u^{*}, X\right\rangle .
$$

If it holds that $\operatorname{dim}\left(\mathcal{Y}_{1} \otimes \cdots \otimes \mathcal{Y}_{n}\right) \leq \operatorname{dim}\left(\mathcal{X}_{1} \otimes \cdots \otimes \mathcal{X}_{n}\right)$, then $\operatorname{dim}\left(\mathcal{Z}_{0}\right) \leq \operatorname{dim}\left(\mathcal{Z}_{n}\right)$, which implies that the inequality in (43) may be taken as an equality for an appropriate choice of a pure state $w$. This completes the proof.

\section{Application to min- and max-entropy}

In this section we connect the main result proved in the previous section to the conditional min- and max-entropy functions. These function, which were first introduced in [6], may be defined as follows. First, one defines the max- and min-relative entropy of $P$ with respect to $Q$, for positive semidefinite operators $P$ and $Q$ (acting on the same space), as follows:

$$
\begin{aligned}
\mathrm{D}_{\max }(P \| Q) & =\log (\min \{\lambda \geq 0: P \leq \lambda Q\}), \\
\mathrm{D}_{\min }(P \| Q) & =-\log \left(\mathrm{F}(P, Q)^{2}\right)
\end{aligned}
$$

Then, with respect to a given state $\rho \in \mathrm{D}(\mathcal{X} \otimes \mathcal{Y})$ of a pair of registers $(\mathrm{X}, \mathrm{Y})$, one defines

$$
\begin{aligned}
& \mathrm{H}_{\min }(\mathrm{X} \mid \mathrm{Y})=-\inf _{\sigma \in \mathrm{D}(\mathcal{Y})} \mathrm{D}_{\max }\left(\rho \| \mathbb{1}_{\mathcal{X}} \otimes \sigma\right), \\
& \mathrm{H}_{\max }(\mathrm{X} \mid \mathrm{Y})=-\inf _{\sigma \in \mathrm{D}(\mathcal{Y})} \mathrm{D}_{\min }\left(\rho \| \mathbb{1}_{\mathcal{X}} \otimes \sigma\right) .
\end{aligned}
$$

It is known that these two quantities are related in the following way: with respect to any pure state $u u^{*}$ of a triple of registers $(\mathrm{X}, \mathrm{Y}, \mathrm{Z})$, one has that

$$
\mathrm{H}_{\min }(\mathrm{X} \mid \mathrm{Y})=-\mathrm{H}_{\max }(\mathrm{X} \mid \mathrm{Z}) \text {. }
$$

(Indeed, in [12] the conditional max-relative entropy of a state of $(\mathbf{X}, \mathbf{Z})$ is defined by the equation (48), which does not depend on which purification of this state is chosen, and is then proved to agree with the definition stated previously.)

Consider any unit vector $u \in \mathcal{X} \otimes \mathcal{Y} \otimes \mathcal{Z}$, which defines a pure state $u u^{*}$ of a triple of registers $(X, Y, Z)$. We will consider two optimization problems defined by $u$, the first of which is as follows:

$$
\begin{aligned}
\operatorname{maximize}: & \left\langle u u^{*}, X\right\rangle \\
\text { subject to: } & X \in \mathcal{S}_{2}(\mathcal{Y}, \mathcal{Z} ; \mathcal{X}, \mathbb{C}) .
\end{aligned}
$$

This optimization problem is illustrated in Figure 5 . In this case, the channel $\Phi_{2}$ takes registers $\mathrm{Z}$ and $\mathrm{W}$ as input and outputs nothing (which is equivalent to outputting the unique state $1 \in \mathrm{D}(\mathbb{C})$ of a one-dimensional system). That is, $\Phi_{2}$ must be the trace mapping. One may therefore simplify this problem, obtaining the following semidefinite program: 


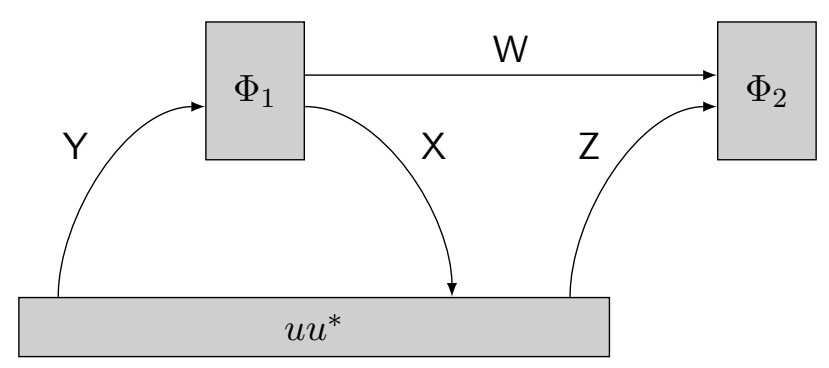

Figure 5: The optimization problem (49) corresponds to a maximization of the linear functions defined by $u u^{*}$ over all strategies given by channels $\Phi_{1}$ and $\Phi_{2}$, for an arbitrary choice of a register W.

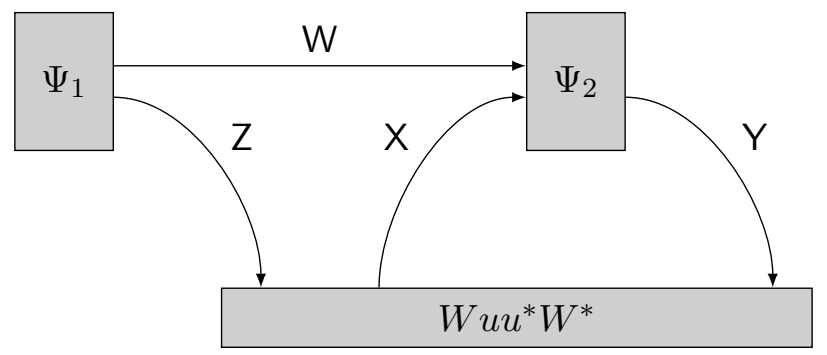

Figure 6: The optimization problem (51) corresponds to a maximization of the linear functions defined by $W u u^{*} W^{*}$ over all strategies given by channels $\Psi_{1}$ and $\Psi_{2}$, for an arbitrary choice of a register $\mathrm{W}$.

\section{Primal problem}

maximize: $\left\langle\operatorname{Tr}_{\mathcal{Z}}\left(u u^{*}\right), X\right\rangle$

subject to: $\operatorname{Tr}_{\mathcal{X}}(X)=\mathbb{1}_{\mathcal{Y}}$, $X \in \operatorname{Pos}(\mathcal{X} \otimes \mathcal{Y})$.
Dual problem

minimize: $\operatorname{Tr}(Y)$

subject to: $\mathbb{1}_{\mathcal{X}} \otimes Y \geq \operatorname{Tr}_{\mathcal{Z}}\left(u u^{*}\right)$,

$Y \in \operatorname{Herm}(\mathcal{X})$.

By examining the dual problem, one sees that the optimal value of this semidefinite program is

$$
2^{-\mathrm{H}_{\min }(\mathrm{X} / \mathrm{Y})}
$$

with respect to the state $u u^{*}$ of $(X, Y, Z)$. König, Renner, and Schaffner [12] observed that the primal problem coincides with the value represented by the expression (50), which is consistent with the observation that strong duality always holds for this semidefinite program (which may be verified through Slater's theorem, for instance).

The second optimization problem we consider is the time-reversal of the first, and may be stated as follows:

$$
\begin{aligned}
\text { maximize: } & \left\langle W u u^{*} W^{*}, Y\right\rangle \\
\text { subject to: } & Y \in \mathcal{S}_{2}(\mathbb{C}, \mathcal{X} ; \mathcal{Z}, \mathcal{Y}) .
\end{aligned}
$$

Figure 6 illustrates the interaction corresponding to this optimization problem. The inclusion $X \in \mathcal{S}_{2}(\mathbb{C}, \mathcal{X} ; \mathcal{Z}, \mathcal{Y})$, for a given operator $X \in \operatorname{Pos}(\mathcal{Z} \otimes \mathcal{Y} \otimes \mathcal{X})$, is equivalent to the condition that $\operatorname{Tr}_{\mathcal{Y}}(X)=\sigma \otimes \mathbb{1}_{\mathcal{X}}$ for some $\sigma \in \mathrm{D}(\mathcal{Z})$. After re-ordering tensor factors, we obtain the following semidefinite program: 


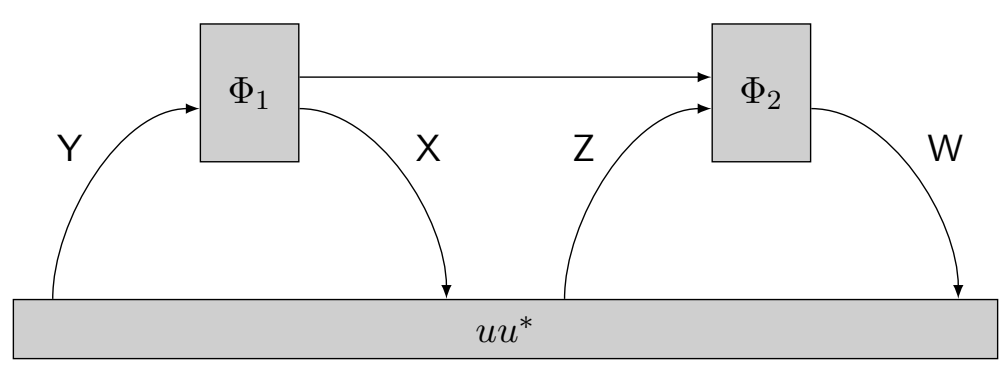

Figure 7: Maximizing the linear function defined by $u u^{*}$ over all four-message strategies of the form depicted yields the left-hand side of (54). By reversing time, the right-hand side of that equation is obtained, and the equality of the two is implied by the main theorem.

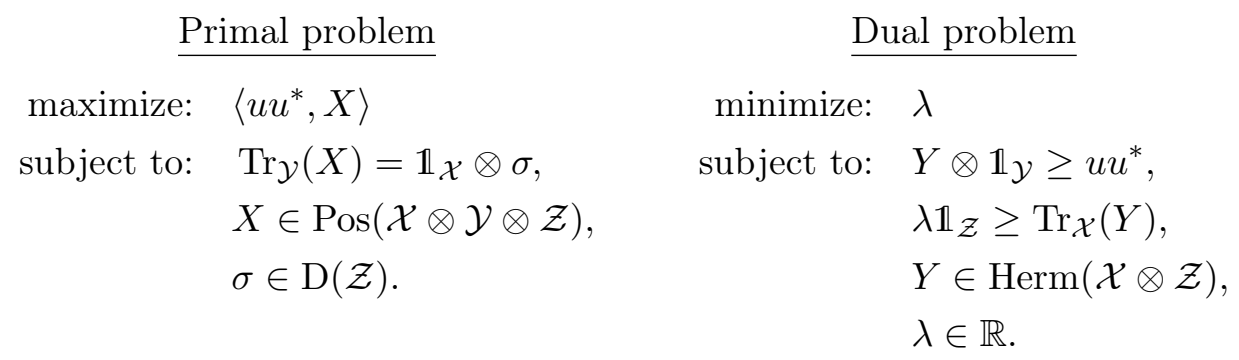

An examination of the primal problem reveals (through Uhlmann's theorem) that the optimal value of this semidefinite program is

$$
2^{\mathrm{H}_{\max }(\mathrm{X} \mid \mathrm{Z})} \text {. }
$$

By our main theorem, it follows that the two optimization problems have the same optimal value, and therefore we obtain an alternative proof that with respect to every pure state of a triple or registers $(\mathrm{X}, \mathrm{Y}, \mathrm{Z})$ one has

$$
\mathrm{H}_{\min }(\mathrm{X} \mid \mathrm{Y})=-\mathrm{H}_{\max }(\mathrm{X} \mid \mathrm{Z}) \text {. }
$$

It is natural to ask if the connections among min-entropy, max-entropy, and optimization problems involving three-message strategies have interesting implications or generalizations for interactions involving four or more messages. As a partial answer to this question, we observe that when our main result is applied to the four-message interaction depicted in Figure 7, it reveals the identity

$$
\max _{\Phi \in \mathrm{C}(\mathcal{Y}, \mathcal{X})} \mathrm{F}\left(\operatorname{Tr}_{\mathcal{W}}\left(u u^{*}\right), J(\Phi) \otimes \mathbb{1}_{\mathcal{Z}}\right)=\max _{\Psi \in \mathrm{C}(\mathcal{W}, \mathcal{Z})} \mathrm{F}\left(\operatorname{Tr}_{\mathcal{X}}\left(u u^{*}\right), \mathbb{1}_{\mathcal{Y}} \otimes J(\Psi)\right)
$$

for all vectors $u \in \mathcal{X} \otimes \mathcal{Y} \otimes \mathcal{Z} \otimes \mathcal{W}$. This identity is appealing in its simplicity and symmetry, and by taking $\mathcal{W}=\mathbb{C}$ (or $\mathcal{Y}=\mathbb{C}$ ) a statement equivalent to (53) for all pure states of $(\mathrm{X}, \mathrm{Y}, \mathrm{Z})$ is obtained. We do not know, however, if the quantity represented by either side of the identity has any direct operational significance.

Other identities may be obtained through a similar methodology, although they become increasingly complex as the number of messages is increased. 


\section{Online pure state entanglement manipulation}

The following three statements are equivalent for a given operator $X \in \mathrm{L}(\mathcal{Y} \otimes \mathcal{X})$ :

1. $X \in \mathcal{S}_{1}(\mathcal{X} ; \mathcal{Y})$. (Equivalently, $X \in \operatorname{Pos}(\mathcal{Y} \otimes \mathcal{X})$ and $\operatorname{Tr}_{\mathcal{Y}}(X)=\mathbb{1}_{\mathcal{X}}$.)

2. $X=\left(\Phi \otimes \mathbb{1}_{\mathrm{L}(\mathcal{X})}\right)\left(\operatorname{vec}\left(\mathbb{1}_{\mathcal{X}}\right) \operatorname{vec}\left(\mathbb{1}_{\mathcal{X}}\right)^{*}\right)$ for some channel $\Phi \in \mathrm{C}(\mathcal{X}, \mathcal{Y})$.

3. $X=\left(\mathbb{1}_{\mathrm{L}(\mathcal{Y})} \otimes \Psi\right)\left(\operatorname{vec}\left(\mathbb{1}_{\mathcal{Y}}\right) \operatorname{vec}\left(\mathbb{1}_{\mathcal{Y}}\right)^{*}\right)$ for some completely positive and unital map $\Psi \in$ $\operatorname{CP}(\mathcal{Y}, \mathcal{X})$.

(Here and throughout this section, vec refers to the vectorization mapping, which is the mapping obtained by extending the transformation $|a\rangle\langle b|\mapsto| a\rangle|b\rangle$ for standard basis states to arbitrary operators by linearity. In particular, $\operatorname{vec}\left(\mathbb{1}_{\mathcal{X}}\right)$ is a non-normalized vector proportional to the canonical maximally entangled pure state corresponding to two identical copies of a system whose state space is $\mathcal{X}$.) The maps $\Phi$ and $\Psi$ uniquely determine one another, and it is reasonable to view these maps as being related by transposition (with respect to the standard basis): $\Psi=\Phi^{\top}$ and $\Phi=\Psi^{\top}$. To obtain a Kraus representation for $\Psi$, for instance, one may simply take a Kraus representation of $\Phi$ and transpose each of the Kraus operators. (The transpose of an arbitrary map can be defined in a manner that is consistent with these statements, but it is sufficient for our needs to focus on channels and completely positive unital maps.)

A generalization of the equivalence mentioned above to the quantum strategy framework may also be verified. For an operator $X \in \mathrm{L}\left(\mathcal{Y}_{1} \otimes \cdots \otimes \mathcal{Y}_{n} \otimes \mathcal{X}_{1} \otimes \cdots \otimes \mathcal{X}_{n}\right)$, these three statements are equivalent:

1. $X \in \mathcal{S}_{n}\left(\mathcal{X}_{1}, \ldots, \mathcal{X}_{n} ; \mathcal{Y}_{1}, \ldots, \mathcal{Y}_{n}\right)$.

2. There exist complex Euclidean spaces $\mathcal{Z}_{1}, \ldots, \mathcal{Z}_{n-1}$ (and $\mathcal{Z}_{0}=\mathbb{C}$ and $\mathcal{Z}_{n}=\mathbb{C}$ ), along with channels $\Phi_{1}, \ldots, \Phi_{n}$ having the form

$$
\Phi_{k} \in \mathrm{C}\left(\mathcal{Z}_{k-1} \otimes \mathcal{X}_{k}, \mathcal{Y}_{k} \otimes \mathcal{Z}_{k}\right),
$$

such that the channel $\Xi_{n} \in \mathrm{C}\left(\mathcal{X}_{1} \otimes \cdots \otimes \mathcal{X}_{n}, \mathcal{Y}_{1} \otimes \cdots \otimes \mathcal{Y}_{n}\right)$ defined as

$$
\Xi_{n}=\left(\mathbb{1}_{\mathrm{L}\left(\mathcal{Y}_{1} \otimes \cdots \otimes \mathcal{Y}_{n-1}\right)} \otimes \Phi_{n}\right) \cdots\left(\Phi_{1} \otimes \mathbb{1}_{\mathrm{L}\left(\mathcal{X}_{2} \otimes \cdots \otimes \mathcal{X}_{n}\right)}\right)
$$

satisfies

$$
X=\left(\Xi_{n} \otimes \mathbb{1}_{\mathrm{L}\left(\mathcal{X}_{1} \otimes \cdots \otimes \mathcal{X}_{n}\right)}\right)\left(\operatorname{vec}\left(\mathbb{1}_{\mathcal{X}_{1} \otimes \cdots \otimes \mathcal{X}_{n}}\right) \operatorname{vec}\left(\mathbb{1}_{\mathcal{X}_{1} \otimes \cdots \otimes \mathcal{X}_{n}}\right)^{*}\right) .
$$

3. There exist complex Euclidean spaces $\mathcal{Z}_{1}, \ldots, \mathcal{Z}_{n-1}$ (and $\mathcal{Z}_{0}=\mathbb{C}$ and $\mathcal{Z}_{n}=\mathbb{C}$ ), along with completely positive and unital maps $\Psi_{1}, \ldots, \Psi_{n}$ having the form

$$
\Psi_{k} \in \mathrm{C}\left(\mathcal{Y}_{k} \otimes \mathcal{Z}_{k}, \mathcal{Z}_{k-1} \otimes \mathcal{X}_{k}\right)
$$

such that the unital map $\Lambda_{n} \in \operatorname{CP}\left(\mathcal{Y}_{1} \otimes \cdots \otimes \mathcal{Y}_{n}, \mathcal{X}_{1} \otimes \cdots \otimes \mathcal{X}_{n}\right)$ defined as

$$
\Lambda_{n}=\left(\Psi_{1} \otimes \mathbb{1}_{\mathrm{L}\left(\mathcal{X}_{2} \otimes \cdots \otimes \mathcal{X}_{n}\right)}\right) \cdots\left(\mathbb{1}_{\mathrm{L}\left(\mathcal{Y}_{1} \otimes \cdots \otimes \mathcal{Y}_{n-1}\right)} \otimes \Psi_{n}\right)
$$

satisfies

$$
X=\left(\mathbb{1}_{\mathrm{L}\left(\mathcal{Y}_{1} \otimes \cdots \otimes \mathcal{Y}_{n}\right)} \otimes \Lambda_{n}\right)\left(\operatorname{vec}\left(\mathbb{1}_{\mathcal{Y}_{1} \otimes \cdots \otimes \mathcal{Y}_{n}}\right) \operatorname{vec}\left(\mathbb{1}_{\mathcal{Y}_{1} \otimes \cdots \otimes \mathcal{Y}_{n}}\right)^{*}\right)
$$


Through this equivalence, for a given state $\rho \in \mathrm{D}\left(\mathcal{Y}_{1} \otimes \cdots \otimes \mathcal{Y}_{n} \otimes \mathcal{X}_{1} \otimes \cdots \otimes \mathcal{X}_{n}\right)$, one arrives at an alternative interpretation of the semidefinite program

$$
\begin{aligned}
\operatorname{maximize}: & \langle\rho, X\rangle \\
\text { subject to: } & X \in \mathcal{S}_{n}\left(\mathcal{X}_{1}, \ldots, \mathcal{X}_{n} ; \mathcal{Y}_{1}, \ldots, \mathcal{Y}_{n}\right)
\end{aligned}
$$

that concerns an online variant of entanglement manipulation, as is explained shortly. The term "online" in this context refers to a situation in which a quantum state must be manipulated in multiple turns, where an output is required immediately after each input system arrives and prior to the next input system being made available, similar to an online process.

By the equivalence of the third statement above to the first, a maximization over all $X \in \mathcal{S}_{n}\left(\mathcal{X}_{1}, \ldots, \mathcal{X}_{n} ; \mathcal{Y}_{1}, \ldots, \mathcal{Y}_{n}\right)$ is equivalent to a maximization over all operators

$$
\left(\mathbb{1}_{\mathrm{L}\left(\mathcal{Y}_{1} \otimes \cdots \otimes \mathcal{Y}_{n}\right)} \otimes \Lambda_{n}\right)\left(\operatorname{vec}\left(\mathbb{1}_{\mathcal{Y}_{1} \otimes \cdots \otimes \mathcal{Y}_{n}}\right) \operatorname{vec}\left(\mathbb{1}_{\mathcal{Y}_{1} \otimes \cdots \otimes \mathcal{Y}_{n}}\right)^{*}\right)
$$

for

$$
\Lambda_{n}=\left(\Psi_{1} \otimes \mathbb{1}_{\mathrm{L}\left(\mathcal{X}_{2} \otimes \cdots \otimes \mathcal{X}_{n}\right)}\right) \cdots\left(\mathbb{1}_{\mathrm{L}\left(\mathcal{Y}_{1} \otimes \cdots \otimes \mathcal{Y}_{n-1}\right)} \otimes \Psi_{n}\right)
$$

and $\Psi_{1}, \ldots, \Psi_{n}$ being completely positive and unital maps of the form

$$
\Psi_{k} \in \mathrm{C}\left(\mathcal{Y}_{k} \otimes \mathcal{Z}_{k}, \mathcal{Z}_{k-1} \otimes \mathcal{X}_{k}\right)
$$

The value of the objective function $\langle\rho, X\rangle$ may therefore be expressed as

$$
\left\langle\left(\mathbb{1}_{\mathrm{L}\left(\mathcal{Y}_{1} \otimes \cdots \otimes \mathcal{Y}_{n}\right)} \otimes \Lambda_{n}^{*}\right)(\rho), \operatorname{vec}\left(\mathbb{1}_{\mathcal{Y}_{1} \otimes \cdots \otimes \mathcal{Y}_{n}}\right) \operatorname{vec}\left(\mathbb{1}_{\mathcal{Y}_{1} \otimes \cdots \otimes \mathcal{Y}_{n}}\right)^{*}\right\rangle,
$$

which is $\operatorname{dim}\left(\mathcal{Y}_{1} \otimes \cdots \otimes \mathcal{Y}_{n}\right)$ times the squared fidelity between the maximally entangled state $\tau \in \mathrm{D}\left(\mathcal{Y}_{1} \otimes \cdots \otimes \mathcal{Y}_{n} \otimes \mathcal{Y}_{1} \otimes \cdots \otimes \mathcal{Y}_{n}\right)$ given by

$$
\tau=\frac{\operatorname{vec}\left(\mathbb{1}_{\mathcal{Y}_{1} \otimes \cdots \otimes \mathcal{Y}_{n}}\right) \operatorname{vec}\left(\mathbb{1}_{\mathcal{Y}_{1} \otimes \cdots \otimes \mathcal{Y}_{n}}\right)^{*}}{\operatorname{dim}\left(\mathcal{Y}_{1} \otimes \cdots \otimes \mathcal{Y}_{n}\right)}
$$

and the state obtained by applying the channel $\Lambda_{n}^{*}$ to the portion of $\rho$ corresponding to the spaces $\mathcal{X}_{1}, \ldots, \mathcal{X}_{n}$. In the case that $n=1$, König, Renner, and Schaffner [12] refer to this quantity as the quantum correlation. This situation is illustrated for the case $n=3$ in Figure 8 .

By Theorem 1, one finds that when $\rho$ is pure, the same optimal value is achieved when the ordering of the channels and the registers on which they act is reversed, as illustrated in Figure 9 for the case $n=3$. That is, when $\rho$ is a pure state, the optimal value of the semidefinite program (61) represents the value

$$
\left\langle\left(\Xi_{n} \otimes \mathbb{1}_{\mathrm{L}\left(\mathcal{X}_{1} \otimes \cdots \otimes \mathcal{X}_{n}\right)}\right)(\rho), \operatorname{vec}\left(\mathbb{1}_{\mathcal{X}_{1} \otimes \cdots \otimes \mathcal{X}_{n}}\right) \operatorname{vec}\left(\mathbb{1}_{\mathcal{X}_{1} \otimes \cdots \otimes \mathcal{X}_{n}}\right)^{*}\right\rangle,
$$

maximized over all channels $\Xi_{n} \in \mathrm{C}\left(\mathcal{Y}_{1} \otimes \cdots \otimes \mathcal{Y}_{n}, \mathcal{X}_{1} \otimes \cdots \otimes \mathcal{X}_{n}\right)$ of the form

$$
\Xi_{n}=\left(\Phi_{1} \otimes \mathbb{1}_{\mathrm{L}\left(\mathcal{X}_{2} \otimes \cdots \otimes \mathcal{X}_{n}\right)}\right) \cdots\left(\mathbb{1}_{\mathrm{L}\left(\mathcal{Y}_{1} \otimes \cdots \otimes \mathcal{Y}_{n-1}\right)} \otimes \Phi_{n}\right)
$$

for channels $\Phi_{1}, \ldots, \Phi_{n}$ taking the form

$$
\Phi_{k} \in \mathrm{C}\left(\mathcal{Y}_{k} \otimes \mathcal{Z}_{k}, \mathcal{Z}_{k-1} \otimes \mathcal{X}_{k}\right)
$$

and for $\mathcal{Z}_{2}, \ldots, \mathcal{Z}_{n-1}$ arbitrary complex Euclidean spaces (along with $\mathcal{Z}_{0}=\mathbb{C}$ and $\mathcal{Z}_{n}=\mathbb{C}$ ). 


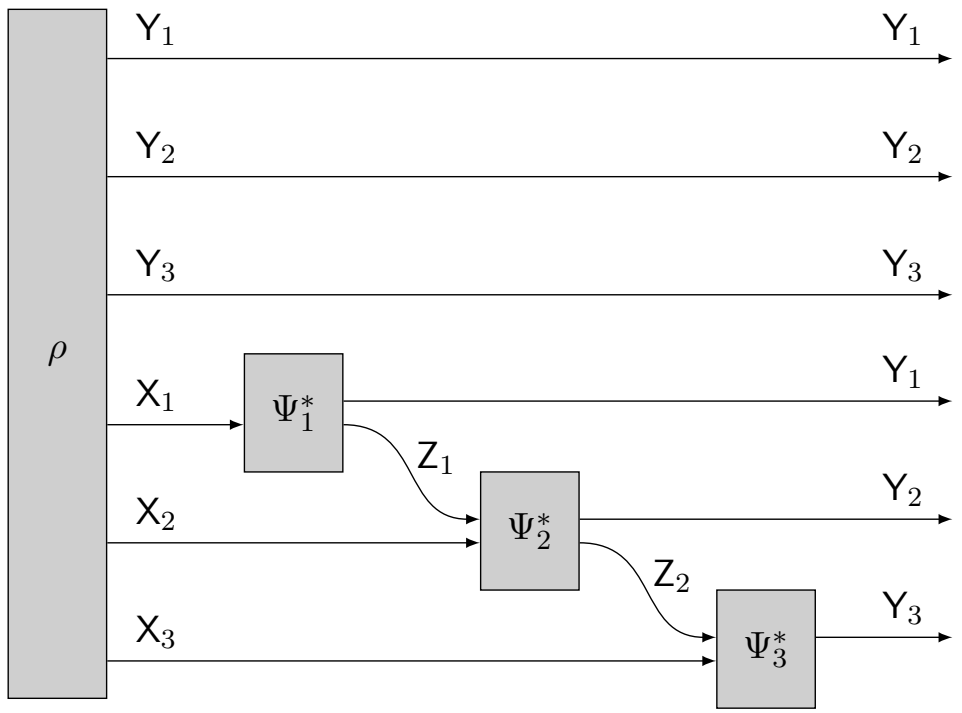

Figure 8: The channel $\Lambda_{3}^{*}=\left(\mathbb{1}_{\mathrm{L}\left(\mathcal{Y}_{1} \otimes \mathcal{Y}_{2}\right)} \otimes \Psi_{3}^{*}\right)\left(\mathbb{1}_{\mathrm{L}\left(\mathcal{Y}_{1}\right)} \otimes \Psi_{2}^{*} \otimes \mathbb{1}_{\mathrm{L}\left(\mathcal{X}_{3}\right)}\right)\left(\Psi_{1}^{*} \otimes \mathbb{1}_{\mathrm{L}\left(\mathcal{X}_{2} \otimes \mathcal{X}_{3}\right)}\right)$ is applied to registers $\left(\mathrm{X}_{1}, \mathrm{X}_{2}, \mathrm{X}_{3}\right)$ of a state $\rho \in \mathrm{D}\left(\mathcal{Y}_{1} \otimes \mathcal{Y}_{2} \otimes \mathcal{Y}_{3} \otimes \mathcal{X}_{1} \otimes \mathcal{X}_{2} \otimes \mathcal{X}_{3}\right)$ with the aim of maximizing the fidelity of the output state with the canonical maximally entangled state.

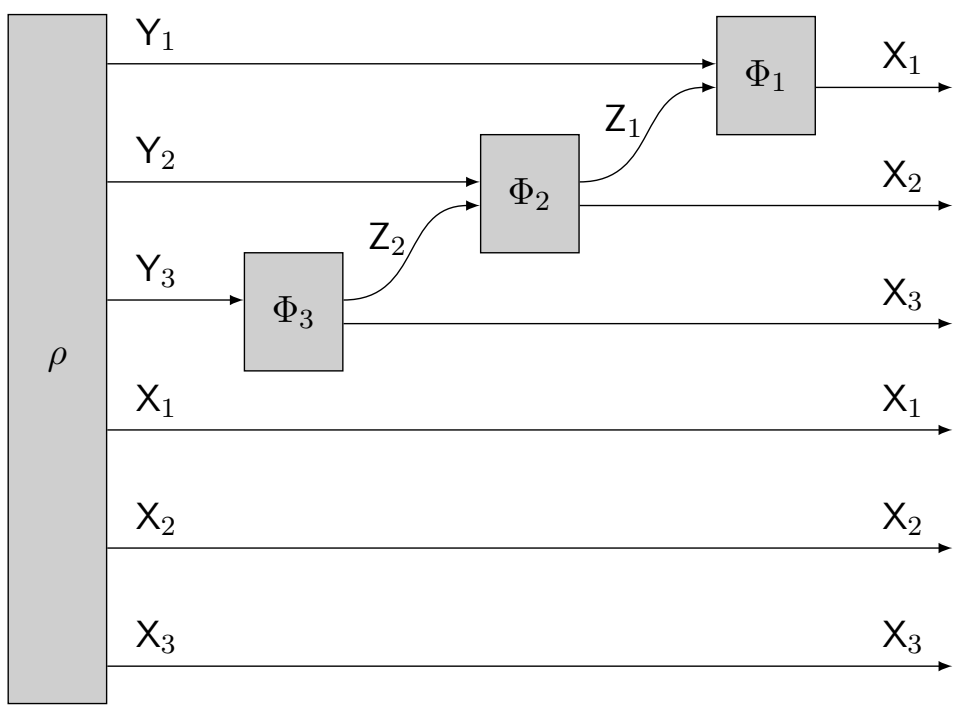

Figure 9: A similar process to the one illustrated in Figure 8, but with channels applied to $Y_{3}, Y_{2}, Y_{1}$ rather than $\mathrm{X}_{1}, \mathrm{X}_{2}, \mathrm{X}_{3}$. 


\section{Conclusion}

We have identified a time-reversal property for rank-one quantum strategy functions, explained its connection to conditional min- and max-entropy, and described an alternative view of this property through an online variant of pure state entanglement manipulation. An obvious question arises: are there interesting applications or implications of this property beyond those we have mentioned?

\section{Acknowledgments}

Yuan Su was supported in part by the Army Research Office (MURI award W911NF-16-10349) and the National Science Foundation (grant 1526380). John Watrous acknowledges the support of Canada's NSERC. We thank Frédéric Dupuis, James R. Garrison, Brian Swingle, Penghui Yao, Ronald de Wolf, and Māris Ozols for helpful discussions, and we thank the anonymous referees for their comments and suggestions.

\section{References}

[1] G. Chiribella and D. Ebler. Optimal quantum networks and one-shot entropies. New Journal of Physics, 18:093053, 2016. DOI: 10.1088/1367-2630/18/9/093053.

[2] G. Chiribella, G. D'Ariano, and P. Perinotti. Quantum circuit architecture. Physical Review Letters, 101(6):060401, 2008. DOI: 10.1103/PhysRevLett.101.060401.

[3] G. Chiribella, G. D'Ariano, and P. Perinotti. Transforming quantum operations: quantum supermaps. Europhysics Letters, 83(3):30004, 2008. DOI: 10.1209/0295-5075/83/30004.

[4] G. Chiribella, G. D'Ariano, and P. Perinotti. Theoretical framework for quantum networks. Physical Review A, 80(2):022339, 2009. DOI: 10.1103/PhysRevA.80.022339.

[5] G. Chiribella, G. D'Ariano, P. Perinotti, D. Schlingemann, and R. Werner. A short impossibility proof of quantum bit commitment. Physics Letters A, 377(15), 2013. DOI: 10.1016/j.physleta.2013.02.045.

[6] N. Datta. Min- and max-relative entropies and a new entanglement monotone. IEEE Transactions on Information Theory, 55(6):2816-2826, 2009. DOI: 10.1109/TIT.2009.2018325.

[7] T. Eggeling, D. Schlingemann, and R. Werner. Semicausal operations are semilocalizable. Europhysics Letters, 57(6):782-788, 2002. DOI: 10.1209/epl/i2002-00579-4.

[8] G. Gutoski. Quantum Strategies and Local Operations. PhD thesis, University of Waterloo, 2009. URI: $10012 / 4903$.

[9] G. Gutoski and J. Watrous. Toward a general theory of quantum games. In Proceedings of the 39th Annual ACM Symposium on Theory of Computing, pages 565-574, 2007. DOI: $10.1145 / 1250790.1250873$.

[10] L. Hardy. The operator tensor formulation of quantum theory. Philosophical Transactions of the Royal Society A, 370(1971):3385-3417, 2012. DOI: 10.1098/rsta.2011.0326.

[11] A. Kitaev, A. Shen, and M. Vyalyi. Classical and Quantum Computation, volume 47 of Graduate Studies in Mathematics. American Mathematical Society, 2002. DOI: $10.1090 / \mathrm{gsm} / 047 / 08$. 
[12] R. König, R. Renner, and C. Schaffner. The operational meaning of min- and maxentropy. IEEE Transactions on Information Theory, 55(9):4337-4347, 2009. DOI: 10.1109/TIT.2009.2025545.

[13] M. Nielsen and I. Chuang. Quantum Computation and Quantum Information. Cambridge University Press, 2000. DOI: 10.1017/CBO9780511976667.

[14] J. Watrous. The Theory of Quantum Information. Cambridge University Press, 2018. DOI: $10.1017 / 9781316848142$.

[15] M. Wilde. Quantum Information Theory. Cambridge University Press, 2013. DOI: 10.1017/CBO9781139525343.

[16] H. Wolkowicz, R. Saigal, and L. Vandenberge, editors. Handbook of Semidefinite Programming: Theory, Algorithms, and Applications. Kluwer Academic Publishers, 2000. DOI: 10.1007/978-1-4615-4381-7. 